\title{
Synthesis and Antimicrobial Activity of Novel Indol Compounds Containing 2-azitidinones and $1,3,4$ Oxadiazoles
}

\author{
J. SREERAMULU* and P. ASHOKGAJAPATHIRAJU \\ Department of Chemistry, S.K.University, Anantapuramu, India. \\ ${ }^{*}$ Corresponding author E-mail: ashok.organic @gmail.com
}

http://dx.doi.org/10.13005/ojc/300234

(Received: March 02, 2014; Accepted: March 29, 2014)

\begin{abstract}
New novel derivatives of 4-(3-(1-((4-acetyl-5-methyl-5-(p-substituted phenyl)-4,5-dihydro1,3,4-oxadiazol-2-yl)methyl)-5-chloro-1H-indol-3-yl)-1-(pyridin-4-yl)-1H-pyrazol-4-yl)-3-chloro-1(4 substituted phenyl)azetidin-2-one (5a-g) were prepared by the condensation of acetohydrazide $(4 a-g)$ with acetic anhydride. The compound $4(a-g)$ was obtained by the reaction of (3) with 4substituted acetophenone in the presence of glacial aceticacid. The synthon (3) was obtained by the reaction of compound(2) with hydrazine hydrate in ethanol. The compound (2) was obtained by the reaction of $(1 \mathrm{H}$-indol-1-yl)acetate $(1)$ with monochloroacetyl chloride in the presence of triethylamine in dioxane. The structure of the newly synthesized compounds were charecterized by IR, NMR, Mass and elemental analysis.
\end{abstract}

Key words: 1,3,4-oxadiazole, acetic anhydride, acetophenone, Monochloro acetyl chloride, antimicrobial activity.

\section{INTRODUCTION}

$1,3,4,-$ Oxadiazoles are five membered heterocycles having two nitrogen atoms and one oxygen atom. 1,3,4-oxadiazoles belong to the group of heterocycles that have been attracting attention for last two decades due to their wide range of biological interactions. Some 1,3,4-oxadiazoles substituted witharyl groups at positions 2 and 5 are of significant interest of polymer and material sciences because of their electro chemical properties(Phosphorescence). 1,3,4-oxadiazole derivatives have played a major role in the pharmaceutical chemistry.Literature reveals that a large number of heterocyclic compounds containing the 1, 3, 4Oxadiazoles ring are associated with diverse pharmacological properties such as antiinflammatory, antimicrobial, fungicidal and antiviral activity[1-4]. Substituted 1,3,4-oxadiazole have revealed antibacterial ${ }^{5,6}$, antitubercular ${ }^{7}$, vasodialatory ${ }^{8}$, antifungal ${ }^{9,10}$, cytotoxic ${ }^{11}$, antiinflammatory and analgesic ${ }^{12-15}$, hypolipidemic ${ }^{16}$, anticancer ${ }^{17,18}$ and ulcerogenic ${ }^{19}$ 
antimycobacterail $^{20}$, anticonvulsant ${ }^{21}$ activities. We have designed and synthesized new 1,3,4Oxadiazoles as a potential antimicrobial agent. The results of this study are discussed in this paper.

\section{MATERIALS AND METHODS}

All the chemicals used in the present investigation were purchased from Ark pharma, Inc. and Sigma-Aldrich Chemicals company, Inc.USA. and used without further purification. TLC was performed on aluminium sheet of silica gel $60 \mathrm{~F}_{254}, \mathrm{E}$ Merk,Germany using iodine as visualizing agent. Melting points were determined in open capillary tubes on Mel-Temp apparatus and are uncorrected (in degree celsius). Columnchromatography was performed on silica gel with different solvent systems as eluents to afford the pure compound. The Infra Red Spectra of the compounds were recorded in $\mathrm{KBr}$ pellets on $\mathrm{FT}-\mathrm{IR}$ (perkin-EImer 1000 units)instrument . All ${ }^{1} \mathrm{H}$ and ${ }^{13} \mathrm{C}-\mathrm{NMR}$ spectra were recorded on a varian $\mathrm{XL}-300$ Spectrometer operating at $400 \mathrm{MHz}$ for ${ }^{1} \mathrm{H}-\mathrm{NMR}$ and $75 \mathrm{MHz}$ for ${ }^{13} \mathrm{C}-\mathrm{NMR}$. The ${ }^{1} \mathrm{H}-\mathrm{NMR}$ spectra were recorded using TMS as an internal standard(Chemical shifts in $\left.\delta_{\mathrm{ppm}}\right)$. The compounds were dissolved in DMSO- $\mathrm{d}_{6}$ and Chemical shifts were referenced to TMS $\left({ }^{1} \mathrm{H}\right.$ and $\left.{ }^{13} \mathrm{C}-\mathrm{NMR}\right)$. Mass spectral data was recorded on FAB-MS instrument at $70 \mathrm{ev}$ with direct inlet system. Elemental analysis were recorded on a Carlo Erba 1108 elemental Analyser, Central Drug Research Institute, Lucknow, India.

\section{Preparation of Intermediates}

2-(5-chloro-3-(4-(3-chloro-4-oxo-1-(4substituted phenyl)azetidin-2-yl)-1- (pyridin-4-yl)-1Hpyrazol-3-yl) - 1 H-indol-1-yl) - N'- (1 - ( psubstitutedphenyl)ethylidene)acetohydrazide (4a-g)

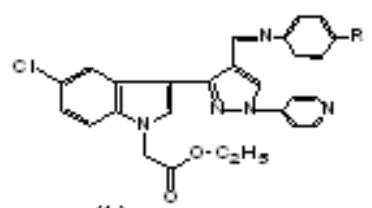

(1)

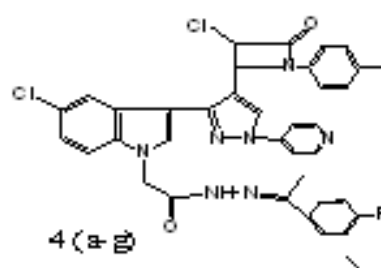
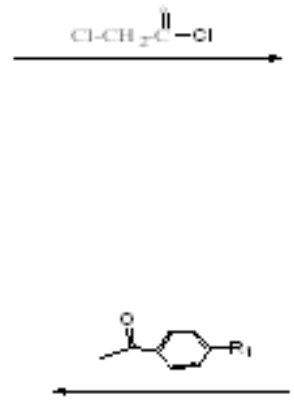

(2)

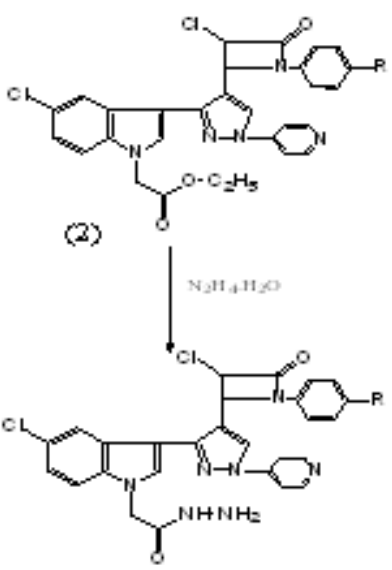

(3)

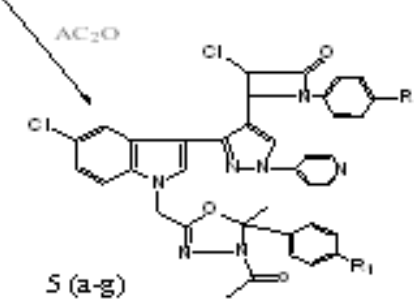

Synthetic route for target compounds

\begin{tabular}{lccccccc}
\hline Compound & $\mathbf{5 a}$ & $\mathbf{5 b}$ & $\mathbf{5 c}$ & $\mathbf{5 d}$ & $\mathbf{5 e}$ & $\mathbf{5 f}$ & $\mathbf{5 g}$ \\
\hline $\mathrm{R} 1$ & $-\mathrm{H}$ & $-\mathrm{CH}_{3}$ & $-\mathrm{OCH}_{3}$ & $4-\mathrm{Cl}$ & $4-\mathrm{Br}$ & $4-\mathrm{NO}_{2}$ & $-\mathrm{CF}_{3}$ \\
$\mathrm{R}$ & $-\mathrm{CF}_{3}$ & $-\mathrm{CF}_{3}$ & $-\mathrm{CF}_{3}$ & $-\mathrm{CF}_{3}$ & $-\mathrm{CF}_{3}$ & $-\mathrm{CF}_{3}$ & $-\mathrm{CF}_{3}$ \\
\hline
\end{tabular}


A mixture of 2-(5-chloro-3-(4-(3-chloro-4oxo-1-(4- substituted phenyl)azetidin-2-yl)-1(pyridin-4-yl)-1H-pyrazol-3-yl)-1H-indol-1$\mathrm{yl})$ acetohydrazide(3) $(1.62 \mathrm{mmol}, 1 \mathrm{~g})$ in hot methanol $(10 \mathrm{ml})$, acetophenone $(10 \mathrm{mmol})$ and a drop of glacial acetic acid were added. Resulting reaction mixture was refluxed for $3 \mathrm{hrs}$ at room temperature.After completion of the reaction as indicated by TLC. The solid that separated was filtered wash with cold methanol and purified by column chromatography by using hexane: ethylacetate $(7: 3)$ used as eluent to afford 2-(5-chloro-3-(4-(3-chloro-4-oxo-1-(4(trifluoromethyl) phenyl) azetidin-2-yl)-1 (pyridin-4-yl)$1 \mathrm{H}$-pyrazol-3-yl)-1H-indol-1-yl)-N'-(1phenylethylidene) acetohydrazide(4a). The reaction procedure leding to (4a), was then extended to $4(b-$ g) from (3) reaction with 4-methyl,4-methoxy,4chloro,4-bromo,4-nitro,4-trifluoromethyl acetophenone to afforded the compounds $4(\mathrm{~b}-\mathrm{g})$.

\section{Synthesis of Compounds}

Ethyl 2-(5-chloro-3-(4-(3-chloro-4-oxo-1-(4substitutedphenyl)azetidin-2-yl)-1- (pyridin-4yl)1H- pyrazol-3-yl)-1H-indol-1-yl)acetate (2)

A mixture of Schiff's Base ethyl2-(5-chloro- 3-(1-(pyridin-4-yl)-4-(((4-(trifluoromethyl) phenyl) imino)methyl)-1H-pyrazol-3-yl)-1 $\mathrm{H}$-indol-1yl)acetate (1) $(20 \mathrm{mmol}, 11.03 \mathrm{~g})$ in acetone, triethylamine $(0.005 \mathrm{~mol}, 0.75 \mathrm{ml})$ was added. To this, a solution of chloroacetyl chloride $(30 \mathrm{mmol}$, $3.5 \mathrm{ml}$ ) was added drop wise with stirring. The Mixture was refluxed up to $8 \mathrm{~h}$. The triethyl amine hydrochloride formed was filtered and washed several times with acetone. The filtrate and washings were mixed and concentrated under reduced pressure. The residue obtained was washed with petroleum ether $\left(40-60^{\circ} \mathrm{C}\right)$ to remove the unreacted Schiff's base and the solid obtained was recrystallized from ethanol to afford compound(2).

2-(5-chloro-3-(4-(3-chloro-4-oxo-1-(4-substituted phenyl)azetidin-2-yl)-1-(pyridin-4-yl)1H -pyrazol-3yl)-1H-indol-1-yl)acetohydrazide (3)

A mixture of Ethyl 2-(5-chloro-3-(4-(3chloro-4-oxo-1-(4-substitutedphenyl)azetidin-2-yl)1- (pyridin-4-yl)-1H-pyrazol-3-yl)-1H-indol-1yl)acetate (2) $(20 \mathrm{mmol})$ and hydrazine hydrate $(30 \mathrm{mmol})$ in ethanol $20 \mathrm{ml}$ was refluxed for $6-7$ hours. The reaction mixture was cooled and poured on to

The structures of the newly synthesized compounds were supported by physical data (Table-1) and following spectral analysis

\begin{tabular}{|c|c|c|c|c|c|c|c|c|c|c|}
\hline \multirow[t]{2}{*}{ Comp } & \multirow[t]{2}{*}{$\mathrm{R}$} & \multirow[t]{2}{*}{$\mathrm{RI}$} & \multirow[t]{2}{*}{ M.P. } & \multirow[t]{2}{*}{$\begin{array}{l}\text { Yield } \\
\text { (\%) }\end{array}$} & \multirow[t]{2}{*}{$\begin{array}{l}\text { Molecular } \\
\text { Formula }\end{array}$} & \multicolumn{4}{|c|}{$\begin{array}{l}\text { Elemental Analysis } \\
\text { Found, Calculated(\%) }\end{array}$} & \multirow[t]{2}{*}{ Rf } \\
\hline & & & & & & $\mathrm{C}(\%)$ & $H(\%)$ & $N(\%)$ & $\mathrm{O}(\%)$ & \\
\hline $5 a$ & $-\mathrm{CF} 3$ & $-H$ & $158-59$ & $65 \%$ & $\mathrm{C}_{38} \mathrm{H}_{28} \mathrm{Cl}_{2} \mathrm{~F}_{3} \mathrm{~N}_{7} \mathrm{O}_{3}$ & $\begin{array}{l}60.02 \\
(60.17)\end{array}$ & $\begin{array}{l}3.67 \\
(3.72)\end{array}$ & $\begin{array}{l}12.78 \\
(12.93)\end{array}$ & $\begin{array}{l}6.17 \\
(6.33)\end{array}$ & 0.60 \\
\hline $5 b$ & $-\mathrm{CF} 3$ & $-\mathrm{CH} 3$ & $152-53$ & $63 \%$ & $\mathrm{C}_{39} \mathrm{H}_{30} \mathrm{Cl}_{2} \mathrm{~F}_{3} \mathrm{~N}_{7} \mathrm{O}_{3}$ & $\begin{array}{l}(60.63) \\
60.47\end{array}$ & $\begin{array}{l}(3.91) \\
3.76\end{array}$ & $\begin{array}{l}(12.69) \\
12.54\end{array}$ & $\begin{array}{l}(6.21) \\
6.04\end{array}$ & 0.62 \\
\hline $5 c$ & $-\mathrm{CF} 3$ & $\mathrm{OCH} 3$ & $142-44$ & $60 \%$ & $\mathrm{C}_{39} \mathrm{H}_{30} \mathrm{Cl}_{2} \mathrm{~F}_{3} \mathrm{~N}_{7} \mathrm{O}_{4}$ & $\begin{array}{l}(59.40) \\
59.24\end{array}$ & $\begin{array}{l}(3.83) \\
3.67\end{array}$ & $\begin{array}{l}(12.43) \\
12.28\end{array}$ & $\begin{array}{l}(8.12) \\
7.98\end{array}$ & 0.72 \\
\hline $5 d$ & $-\mathrm{CF} 3$ & $4-\mathrm{Cl}$ & $165-67$ & $62 \%$ & $\mathrm{C}_{38} \mathrm{H}_{27} \mathrm{Cl}_{3} \mathrm{~F}_{3} \mathrm{~N}_{7} \mathrm{O}_{3}$ & $\begin{array}{l}(57.55) \\
57.40\end{array}$ & $\begin{array}{l}(3.43) \\
3.28\end{array}$ & $\begin{array}{l}(12.36) \\
12.21\end{array}$ & $\begin{array}{l}(6.05) \\
5.90\end{array}$ & 0.68 \\
\hline $5 e$ & $-\mathrm{CF} 3$ & $4-\mathrm{Br}$ & $162-63$ & $63 \%$ & $\mathrm{C}_{38} \mathrm{H}_{27} \mathrm{BrCl}_{2} \mathrm{~F}_{3} \mathrm{~N}_{7} \mathrm{O}_{3}$ & $\begin{array}{l}(54.50) \\
54.35\end{array}$ & $\begin{array}{l}(3.25) \\
3.10\end{array}$ & $\begin{array}{l}(11.71) \\
11.56\end{array}$ & $\begin{array}{l}(5.73) \\
5.57\end{array}$ & 0.63 \\
\hline $5 f$ & $-\mathrm{CF} 3$ & 4-NO2 & 2184-86 & $70 \%$ & $\mathrm{C}_{38} \mathrm{H}_{27} \mathrm{Cl}_{2} \mathrm{~F}_{3} \mathrm{~N}_{8} \mathrm{O}_{5}$ & $\begin{array}{l}(56.80) \\
56.65\end{array}$ & $\begin{array}{l}(3.39) \\
3.23\end{array}$ & $\begin{array}{l}(13.94) \\
13.77\end{array}$ & $\begin{array}{l}(9.96) \\
9.80\end{array}$ & 0.48 \\
\hline $5 g$ & $-\mathrm{CF} 3$ & 4-CF3 & $178-79$ & $68 \%$ & $\mathrm{C}_{39} \mathrm{H}_{27} \mathrm{Cl}_{2} \mathrm{~F}_{6} \mathrm{~N}_{7} \mathrm{O}_{3}$ & $\begin{array}{l}(56.67) \\
56.52\end{array}$ & $\begin{array}{l}(3.29) \\
3.13\end{array}$ & $\begin{array}{l}(11.86) \\
11.70\end{array}$ & $\begin{array}{l}(5.81) \\
5.65\end{array}$ & 0.54 \\
\hline
\end{tabular}


ice cold water with stirring. The progress of the reaction was monitored by TLC with hexane:ethyl acetate(7:3) as elutent. The separated solid was filtered, washed with water and recrystallized from ethanol to afford compound (3).

4-(3-(1-)((4-acetyl-5-methyl-5-(p-substituted phenyl)-4,5-dihydro-1,3,4-oxadiazol-2-yl) methyl)5-chloro-1H-indol-3-yl)-1-(pyridin-4-yl)-1Hpyrazol-4-yl)-3-chloro-1-(4-substitu ted phenyl)azetidin-2-one (5a-g)

A mixture of $4 \mathrm{a}(1 \mathrm{mmol}, 716.54 \mathrm{mg})$ and excessive acetic anhydride $(10 \mathrm{ml})$ was refluxed for $3 \mathrm{hrs}$. The progress of the reaction was monitored by TLC. The excessive acetic anhydride was distilled off and the residue was poured on to crushed ice. The solid thus obtained was filtered and purified by column chromatography by using hexane: ethylacetate (7:3) as eluent to afford compound(5a) (493.07 $\mathrm{mg}, 0.65 \mathrm{mmol})$. The above cyclisation reaction was then extended to synthesize $5(\mathrm{~b}-\mathrm{g})$ from $4(\mathrm{~b}-\mathrm{g})$ reaction with acetic anhydride.

\section{RESULTS AND DISCUSSION}

The target compounds were synthesized via the route as shown in the Scheme.The synthon required for the synthesis of the target molecules was prepared by a reported method, filtered and recrystallized from ethanol. For all the synthesized compounds, the progress of the reaction was monitored by TLC with cyclohexane, ethylacetate $(7: 3)$ as mobile phase. All the synthesized structures showed satisfactory result. The chemical shift values of the synthesized compounds were full agreement with the number of protons present in it.

Physical, Analytical and Spectral data for the synthesized title compounds are given as follows. Characterization of 2-(5-chloro-3-(4-(3chloro-4-oxo-1-(4-(trifluoromethyl)phenyl) azetiding-2-yl)-1-(pyridin-4-yl)-1H-pyrazol-3-yl)-1Hindol-1-yl)-N'-(1phenylethylidene) acetohydrazide (4a) yield $65 \%$, M.P: $162-63^{\circ} \mathrm{C}, \mathrm{IR}(\mathrm{KBR}):(\delta \mathrm{ppm})$

Table 2:

\begin{tabular}{|c|c|c|c|c|c|c|c|c|c|c|}
\hline \multirow[t]{2}{*}{ Comp } & \multirow[t]{2}{*}{$\mathbf{R}$} & \multirow[t]{2}{*}{$\mathbf{R}^{\prime}$} & \multirow[t]{2}{*}{ M.P. } & \multirow[t]{2}{*}{$\begin{array}{l}\text { Yield } \\
\text { (\%) }\end{array}$} & \multirow[t]{2}{*}{$\begin{array}{l}\text { Molecular } \\
\text { Formula }\end{array}$} & \multicolumn{4}{|c|}{$\begin{array}{l}\text { Elemental Analysis } \\
\text { Found, Calculated(\%) }\end{array}$} & \multirow[t]{2}{*}{ Rf } \\
\hline & & & & & & C(\%) & $H(\%)$ & N (\%) & $\mathrm{O}(\%)$ & \\
\hline $4 a$ & $4-\mathrm{CF}_{3}$ & $-H$ & $162-63$ & $65 \%$ & $\mathrm{C}_{36} \mathrm{H}_{26} \mathrm{Cl}_{2} \mathrm{~F}_{3} \mathrm{~N}_{7} \mathrm{O}_{2}$ & $\begin{array}{l}60.15 \\
(60.34)\end{array}$ & $\begin{array}{c}3.41 \\
(3.66)\end{array}$ & $\begin{array}{r}13.57 \\
(13.68)\end{array}$ & $\begin{array}{c}4.25 \\
(4.47)\end{array}$ & 0.52 \\
\hline $4 b$ & $4-\mathrm{CF}_{3}$ & $-\mathrm{CH}_{3}$ & $157-58$ & $62 \%$ & $\mathrm{C}_{37} \mathrm{H}_{28} \mathrm{Cl}_{2} \mathrm{~F}_{3} \mathrm{~N}_{7} \mathrm{O}_{2}$ & $\begin{array}{c}60.62 \\
(60.83)\end{array}$ & $\begin{array}{r}3.54 \\
(3.86)\end{array}$ & $\begin{array}{r}13.21 \\
(13.42)\end{array}$ & $\begin{array}{c}4.17 \\
(4.38)\end{array}$ & 0.57 \\
\hline $4 c$ & $4-\mathrm{CF}_{3}$ & $\mathrm{OCH}_{3}$ & $148-49$ & $60 \%$ & $\mathrm{C}_{37} \mathrm{H}_{28} \mathrm{Cl}_{2} \mathrm{~F}_{3} \mathrm{~N}_{7} \mathrm{O}_{3}$ & $\begin{array}{c}59.34 \\
(59.53)\end{array}$ & $\begin{array}{c}3.57 \\
(3.78)\end{array}$ & $\begin{array}{c}12.95 \\
(13.13)\end{array}$ & $\begin{array}{c}6.26 \\
(6.43)\end{array}$ & 0.65 \\
\hline $4 d$ & $4-\mathrm{CF}_{3}$ & $4-\mathrm{Cl}$ & $168-69$ & $63 \%$ & $\mathrm{C}_{36} \mathrm{H}_{25} \mathrm{Cl}_{3} \mathrm{~F}_{3} \mathrm{~N}_{7} \mathrm{O}_{2}$ & $\begin{array}{c}57.42 \\
(57.58)\end{array}$ & $\begin{array}{l}3.18 \\
(3.36)\end{array}$ & $\begin{array}{c}12.88 \\
(13.06)\end{array}$ & $\begin{array}{c}4.07 \\
(4.26)\end{array}$ & 0.53 \\
\hline $4 e$ & $4-\mathrm{CF}_{3}$ & $4-\mathrm{Br}$ & $165-67$ & $64 \%$ & $\mathrm{C}_{36} \mathrm{H}_{25} \mathrm{BrCl}_{2} \mathrm{~F}_{3} \mathrm{~N}_{7} \mathrm{O}_{2}$ & $\begin{array}{l}54.16 \\
(54.36)\end{array}$ & $\begin{array}{c}3.02 \\
(3.17)\end{array}$ & $\begin{array}{c}12.15 \\
(12.33)\end{array}$ & $\begin{array}{c}3.83 \\
(4.02)\end{array}$ & 0.55 \\
\hline $4 f$ & $4-\mathrm{CF}_{3}$ & $4-\mathrm{NO}_{2}$ & $186-87$ & $70 \%$ & $\mathrm{C}_{36} \mathrm{H}_{25} \mathrm{Cl}_{2} \mathrm{~F}_{3} \mathrm{~N}_{8} \mathrm{O}_{4}$ & $\begin{array}{l}56.63 \\
(56.78)\end{array}$ & $\begin{array}{c}3.16 \\
(3.31)\end{array}$ & $\begin{array}{c}14.58 \\
(14.71)\end{array}$ & $\begin{array}{c}8.24 \\
(8.40)\end{array}$ & 0.46 \\
\hline $4 g$ & $4-\mathrm{CF} 3$ & 4-CF3 & $175-76$ & $68 \%$ & $\mathrm{C}_{37} \mathrm{H}_{25} \mathrm{Cl}_{2} \mathrm{~F}_{6} \mathrm{~N}_{7} \mathrm{O}_{2}$ & $\begin{array}{c}56.48 \\
(56.64)\end{array}$ & $\begin{array}{c}3.07 \\
(3.21)\end{array}$ & $\begin{array}{c}12.35 \\
(12.50)\end{array}$ & $\begin{array}{c}3.92 \\
(4.08)\end{array}$ & 0.49 \\
\hline
\end{tabular}


$3190 \mathrm{~cm}^{-1}(-\mathrm{NH}), 3041 \mathrm{~cm}^{-1}(=\mathrm{CH}), 1696 \mathrm{~cm}^{-1}(\mathrm{C}=\mathrm{O})$, $1625 \mathrm{~cm}^{-1}(\mathrm{C}=\mathrm{N}), 677 \mathrm{~cm}^{-1}(\mathrm{C}-\mathrm{Cl})$ respectively. ${ }^{1} \mathrm{H}-$ NMR (400MHz, DMSO-d $)_{6}$ 'ppm: 10.90(s,1H,$\mathrm{CONH}), 8.10(\mathrm{~s}, 1 \mathrm{H}$, Pyrazole),7.75-8.40(m,4H of $\left.\mathrm{C}_{5} \mathrm{H}_{4} \mathrm{~N}\right)$ 7.30-7.70(m,4H,-CH of indol),6.80$7.20\left(\mathrm{~m}, 9 \mathrm{H}\right.$, of $-\mathrm{C}_{6} \mathrm{H}_{5}$ and $\left.\mathrm{C}_{6} \mathrm{H}_{4} \mathrm{CF}_{3}\right), 5.43(\mathrm{~d}, 1 \mathrm{H},-\mathrm{CH}$ of azitidin attached to $-\mathrm{Cl}), 5.10(\mathrm{~d}, 1 \mathrm{H}, \mathrm{CH}$ of azitidin ring),3.65 (s,2H, N-CH $-\mathrm{CO}), 2.25\left(\mathrm{~s}, 3 \mathrm{H}, \mathrm{N}-\mathrm{CH}_{3}\right)$. $\operatorname{Mass}(\mathrm{m} / \mathrm{z})$ : 715.15 , Anal. Calcd. For $\mathrm{C}_{28} \mathrm{H}_{20} \mathrm{Cl}_{2} \mathrm{~F}_{3} \mathrm{~N}_{7} \mathrm{O}_{2}$ : C, 60.34\%; H, 3.66\%; N, 13.68\%; O, $4.47 \%$. Found: C $60.15 \%$, H $3.41 \%$, N 13.57\%, O $4.25 \%$.

Characterization of 2-(5-chloro-3-(4-(3-chloro4-oxo-1-(4-(trifluoromethyl)phenyl) azitidin -2-yl)1-(pyridin-4-yl)-1H-pyrazol-3-yl)-1H-indol-1-yl)N'(1-(p-tolyl)ethylidene) acetohydra zide (4b)

Yield $60 \%$, M.P: $157-58{ }^{\circ} \mathrm{C}$, IR (KBR) : ('ppm) $3185 \mathrm{~cm}^{-1}(-\mathrm{NH}), 3042 \mathrm{~cm}^{-1}(=\mathrm{CH}), 1685 \mathrm{~cm}^{-}$ ${ }^{1}(\mathrm{C}=\mathrm{O}), \quad 1625 \mathrm{~cm}^{-1}(\mathrm{C}=\mathrm{N}), \quad 676 \mathrm{~cm}^{-1}(\mathrm{C}-\mathrm{Cl})$ respectively. ${ }^{1} \mathrm{H}-\mathrm{NMR}\left(400 \mathrm{MHz}, \mathrm{DMSO}-\mathrm{d}_{6}\right)$ 'ppm: 10.89 (s,1H,-CONH),8.09(s,1H,Pyrazole), 7.75$8.40\left(\mathrm{~m}, 4 \mathrm{H}\right.$ of $\left.-\mathrm{C}_{5} \mathrm{H}_{4} \mathrm{~N}\right), 7.30-7.70(\mathrm{~m}, 4 \mathrm{H},-\mathrm{CH}$ of indol), $6.85-7.15\left(\mathrm{~m}, 8 \mathrm{H}\right.$, of $-\mathrm{C}_{6} \mathrm{H}_{4}$ and $\left.\mathrm{C}_{6} \mathrm{H}_{4} \mathrm{CF}_{3}\right)$ $, 5.42(\mathrm{~d}, 1 \mathrm{H},-\mathrm{CH}$ of azitidin attached to $-\mathrm{Cl}), 5.11$ $(\mathrm{d}, 1 \mathrm{H}, \mathrm{CH}$ of azitidin ring),3.60(s,2H, N-CH $-\mathrm{CO})$, 2.30(s,3H,N-CH$)_{3}, 1.52\left(\mathrm{~s}, 1 \mathrm{H},-\mathrm{CH}_{3}\right)$.

Mass(m/z) : 729.16, Anal.Calcd.For $\mathrm{C}_{37} \mathrm{H}_{28} \mathrm{Cl}_{2} \mathrm{~F}_{3} \mathrm{~N}_{7} \mathrm{O}_{2}$ : C, 60.83\%; H, 3.86\%; N, 13.42\%; O, 4.38\%. Found: C 60.62\%, H 3.54\%, N 13.21\%, O $4.17 \%$.

Characterization of 2-(5-chloro-3-(4-(3-chloro-4oxo-1-(4-(trifluoromethyl)phenyl) azetidin-2-yl)-1(pyridine-4-yl)-1H-pyrazol-3-yl)-1H-indol-1-yl)N'(1-(4-methoxyphenyl) ethylidene ) acetohydrazide (4c)

yield $63 \%$, M.P: $148-49{ }^{\circ} \mathrm{C}$, IR (KBR) : ('Àppm) $3184 \mathrm{~cm}^{-1}(-\mathrm{NH}), 3040 \mathrm{~cm}^{-1}(=\mathrm{CH}), 1680 \mathrm{~cm}^{-}$ ${ }^{1}(\mathrm{C}=\mathrm{O}), \quad 1620 \mathrm{~cm}^{-1}(\mathrm{C}=\mathrm{N}), \quad 675 \mathrm{~cm}^{-1}(\mathrm{C}-\mathrm{Cl})$ respectively. ${ }^{1} \mathrm{H}-\mathrm{NMR}\left(400 \mathrm{MHz}\right.$,DMSO-d $\left.{ }_{6}\right)$

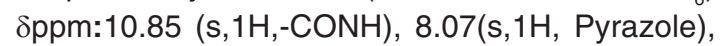
7.75-8.40(m,4 $\mathrm{H}$ of $\left.-\mathrm{C}_{5} \mathrm{H}_{4} \mathrm{~N}\right), 7.30-7.70(\mathrm{~m}, 4 \mathrm{H},-\mathrm{CH}$ of indol),6.80-7.16(m,8 $\mathrm{H}$, of $-\mathrm{C}_{6} \mathrm{H}_{4}$ and $\left.\mathrm{C}_{6} \mathrm{H}_{4} \mathrm{CF}_{3}\right)$ $5.40(\mathrm{~d}, 1 \mathrm{H},-\mathrm{CH}$ of azitidin attached to $-\mathrm{Cl}), 5.10$ (d, $1 \mathrm{H}, \mathrm{CH}$ of azitidin ring),3.55(s,2 H, N-CH CO), 2.32(s,3H, N-CH $), 3.85\left(\mathrm{~s}, 1 \mathrm{H},-\mathrm{OCH}_{3}\right) \cdot \operatorname{Mass}(\mathrm{m} /$ z) : 745.16, Anal.Calcd.For $\mathrm{C}_{37} \mathrm{H}_{28} \mathrm{Cl}_{2} \mathrm{~F}_{3} \mathrm{~N}_{7} \mathrm{O}_{3}: \mathrm{C}$, 59.53\%; H, 3.78\%; N, 13.13\%; O, 6.43\%. Found: C
$59.34 \%$, H $3.57 \%$, N $12.95 \%$, O 6.26\%.

Characterization of 2-(5-chloro-3-(4-(3-chloro-4oxo-1-(4-(trifluoromethyl)phenyl) azetidin -2-yl)1-(pyridin-4-yl)-1H-pyrazol-3-yl)-1H-indol-1-yl)N'-(1-(4-chlorophenyl) ethylidene) acetohydrazide (4d)

yield $67 \%$, M.P: $168-69^{\circ} \mathrm{C}$, IR $(\mathrm{KBR}):(\delta$ ppm) $3200 \mathrm{~cm}^{-1}(-\mathrm{NH}), 3045 \mathrm{~cm}^{-1}(=\mathrm{CH}), 1690 \mathrm{~cm}$ ${ }^{1}(\mathrm{C}=\mathrm{O}), \quad 1623 \mathrm{~cm}^{-1}(\mathrm{C}=\mathrm{N}), \quad 676 \mathrm{~cm}^{-1}(\mathrm{C}-\mathrm{Cl})$ respectively. ${ }^{1} \mathrm{H}-\mathrm{NMR}\left(400 \mathrm{MHz}, \mathrm{DMSO}-\mathrm{d}_{6}\right) \delta \mathrm{ppm}$ : $10.92(\mathrm{~s}, 1 \mathrm{H},-\mathrm{CONH}), 8.08(\mathrm{~s}, 1 \mathrm{H}, \mathrm{Pyrazole}), 7.75$ $8.40\left(\mathrm{~m}, 4 \mathrm{H}\right.$ of $\left.-\mathrm{C}_{5} \mathrm{H}_{4} \mathrm{~N}\right), 7.30-7.70(\mathrm{~m}, 4 \mathrm{H},-\mathrm{CH}$ of indol), 6.85-7.18 (m,8H, of $-\mathrm{C}_{6} \mathrm{H}_{4} \mathrm{Cl}$ and $\left.\mathrm{C}_{6} \mathrm{H}_{4} \mathrm{CF}_{3}\right)$ $5.44(\mathrm{~d}, 1 \mathrm{H},-\mathrm{CH}$ of azitidin attached to $-\mathrm{Cl}), 5.10$ $\left(\mathrm{d}, 1 \mathrm{H}, \mathrm{CH}\right.$ of azitidin ring), $3.57\left(\mathrm{~s}, 2 \mathrm{H}, \mathrm{N}-\mathrm{CH}_{2}-\right.$ CO),2.31(s,3H, N-CH$)$. Mass(m/z) : 749.11, Anal. Calcd.For $\mathrm{C}_{36} \mathrm{H}_{25} \mathrm{Cl}_{3} \mathrm{~F}_{3} \mathrm{~N}_{7} \mathrm{O}_{2}$ : C, 57.58\%; H, 3.36\%; N, $13.06 \%$; O, $4.26 \%$, Found: C 59.42\%, H $3.18 \%$, N $12.88 \%$, O $4.01 \%$.

Characterization of N'-(1-(4-bromophenyl) ethylidene)-2-(5-chloro-3-(4-(3-chloro-4-oxo-1-(4(trifluoromethyl)phenyl)azetidin-2-yl)-1-(pyridin4-yl) - 1 H-pyrazol-3-yl) - 1 H-indol-1-yl) acetohydrazide (4e)

yield $66 \%$, M.P: $165-67^{\circ} \mathrm{C}$, IR (KBR) :('Àppm) $3205 \mathrm{~cm}^{-1}(-\mathrm{NH}), 3044 \mathrm{~cm}^{-1}(=\mathrm{CH}), 1687 \mathrm{~cm}^{-1}$ $(\mathrm{C}=\mathrm{O}), 1623 \mathrm{~cm}^{-1}(\mathrm{C}=\mathrm{N}), 677 \mathrm{~cm}^{-1}(\mathrm{C}-\mathrm{Cl})$ respectively.

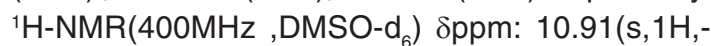
$\mathrm{CONH}), 8.09(\mathrm{~s}, 1 \mathrm{H}$, Pyrazole), $7.75-8.40(\mathrm{~m}, 4 \mathrm{H}$ of $\left.\mathrm{C}_{5} \mathrm{H}_{4} \mathrm{~N}\right), 7.30-7.70(\mathrm{~m}, 4 \mathrm{H},-\mathrm{CH}$ of indol $), 6.80$ $7.20\left(\mathrm{~m}, 8 \mathrm{H}\right.$, of $-\mathrm{C}_{6} \mathrm{H}_{4} \mathrm{Br}$ and $\left.\mathrm{C}_{6} \mathrm{H}_{4} \mathrm{CF}_{3}\right) 5.43(\mathrm{~d}, 1 \mathrm{H},-\mathrm{CH}$ of azitidin attached to $-\mathrm{Cl}), 5.10(\mathrm{~d}, 1 \mathrm{H}, \mathrm{CH}$ of azitidin ring), $3.58\left(\mathrm{~s}, 2 \mathrm{H}, \mathrm{N}-\mathrm{CH}_{2}-\mathrm{CO}\right), 2.32\left(\mathrm{~s}, 3 \mathrm{H}, \mathrm{N}-\mathrm{CH}_{3}\right)$. $\operatorname{Mass}(\mathrm{m} / \mathrm{z}): 793.06$, Anal.Calcd.For $\mathrm{C}_{36} \mathrm{H}_{25}$ $\mathrm{BrCl}_{2} \mathrm{~F}_{3} \mathrm{~N}_{7} \mathrm{O}_{2}$ : C, 54.36\%; H, 3.17\%; N, 12.33\%; O, $4.02 \%$. Found: C 59.16\%, H $3.02 \%$, N $12.15 \%$, O $3.83 \%$.

Characterization of 2-(5-chloro-3-(4-(3-chloro-4oxo-1-(4-(trifluoromethyl)phenyl) azetidin-2-yl)-1(pyridin-4-yl)-1H-pyrazol-3-yl)-1H-indol-1-yl)-N'(1-(4-nitrophenyl) ethylidene)acetohydrazide (4f) yield $68 \%$, M.P: $186-87^{\circ} \mathrm{C}$, IR (KBR):

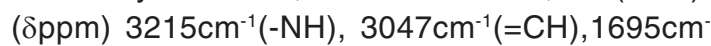
${ }^{1}(\mathrm{C}=\mathrm{O}) \quad, 1626 \mathrm{~cm}^{-1}(\mathrm{C}=\mathrm{N}), \quad 678 \quad \mathrm{~cm}^{-1}(\mathrm{C}-\mathrm{Cl})$ respectively. ${ }^{1} \mathrm{H}-\mathrm{NMR}\left(400 \mathrm{MHz}\right.$,DMSO- $\left.\mathrm{d}_{6}\right)$ Sppm:10.95 (s, $1 \mathrm{H},-$ $\mathrm{CONH}), 8.10(\mathrm{~s}, 1 \mathrm{H}$, Pyrazole), $7.75-8.40(\mathrm{~m}, 4 \mathrm{H}$ of - 
$\left.\mathrm{C}_{5} \mathrm{H}_{4} \mathrm{~N}\right), 7.30-7.70(\mathrm{~m}, 4 \mathrm{H},-\mathrm{CH}$ of indol) ,6.95$7.28\left(\mathrm{~m}, 8 \mathrm{H}, \mathrm{of}-\mathrm{C}_{6} \mathrm{H}_{4} \mathrm{NO}_{2}\right.$ and $\left.\mathrm{C}_{6} \mathrm{H}_{4} \mathrm{CF}_{3}\right), 5.44(\mathrm{~d}, 1 \mathrm{H},-$ $\mathrm{CH}$ of azitidin attached to $-\mathrm{Cl}), 5.09(\mathrm{~d}, 1 \mathrm{H}, \mathrm{CH}$ of azitidin ring), $3.69\left(\mathrm{~s}, 2 \mathrm{H}, \mathrm{N}-\mathrm{CH}_{2}-\mathrm{CO}\right), 2.30(\mathrm{~s}, 3 \mathrm{H}, \mathrm{N}-$ $\left.\mathrm{CH}_{3}\right)$. Mass $(\mathrm{m} / \mathrm{z}): 760.13$, Anal.Calcd.For $\mathrm{C}_{36} \mathrm{H}_{25} \mathrm{Cl}_{2} \mathrm{~F}_{3} \mathrm{~N}_{8} \mathrm{O}_{4}$ : C, 56.78\%; H, 3.31\%; N, $14.71 \%$; O, $8.40 \%$. Found: C 56.63\%, H $3.16 \%$, N $14.58 \%$, O $8.24 \%$.

Characterization of 2-(5-chloro-3-(4-(3-chloro-4oxo-1-(4-(trifluoromethyl)phenyl) azetidin-2-yl)-1(pyridine-4-yl)-1H-pyrazol-3-yl)-1H-indol-1-yl)-N'(1(4(trifluoromethyl) phenyl) ethylidene) acetohydrazide $(4 \mathrm{~g})$

yield $70 \%$, M.P: $175-76{ }^{\circ} \mathrm{C}, \mathrm{IR}(\mathrm{KBR}):(\delta \mathrm{ppm})$ $3210 \mathrm{~cm}^{-1}(-\mathrm{NH}), 3046 \mathrm{~cm}^{-1}(=\mathrm{CH}), 1694 \mathrm{~cm}^{-}$ ${ }^{1}(\mathrm{C}=\mathrm{O}), 1624 \mathrm{c} \mathrm{m}^{-1}(\mathrm{C}=\mathrm{N}), 676 \mathrm{c} \mathrm{m}^{-1}(\mathrm{C}-$ Cl)respectively. ${ }^{1} \mathrm{H}-\mathrm{NMR}\left(400 \mathrm{MHz}\right.$, DMSO-d $\left.\mathrm{d}_{6}\right) \delta \mathrm{ppm}$ :10.93 (s,1H,-CONH), 8.081(s,1H,Pyrazole), 7.75$8.40\left(\mathrm{~m}, 4 \mathrm{H}\right.$ of $\left.-\mathrm{C}_{5} \mathrm{H}_{4} \mathrm{~N}\right), 7.30-7.70(\mathrm{~m}, 4 \mathrm{H},-\mathrm{CH}$ of indol), $6.90-7.25\left(\mathrm{~m}, 8 \mathrm{H}\right.$, of two $-\mathrm{C}_{6} \mathrm{H}_{4} \mathrm{CF}_{3}$ rings), $5.45(\mathrm{~d}, 1 \mathrm{H},-\mathrm{CH}$ of azitidin attached to $-\mathrm{Cl}), 5.11$ $\left(\mathrm{d}, 1 \mathrm{H}, \mathrm{CH}\right.$ of azitidin ring), $3.65\left(\mathrm{~s}, 2 \mathrm{H}, \mathrm{N}-\mathrm{CH}_{2}-\right.$ CO), 2.35(s,3H, N-CH ${ }_{3}$ ). $\operatorname{Mass}(\mathrm{m} / \mathrm{z}): 783.14$, Anal.Calcd.For $\mathrm{C}_{37} \mathrm{H}_{25} \mathrm{Cl}_{2} \mathrm{~F}_{6} \mathrm{~N}_{7} \mathrm{O}_{2}: \mathrm{C}, 56.64 \% ; \mathrm{H}$, $3.21 \%$; N $12.50 \%$; O, $4.08 \%$. Found: C $56.48 \%, \mathrm{H}$ $3.07 \%, \mathrm{~N} 12.35 \%$, O $3.92 \%$.

Characterization of 4-(3-(1-((4-acetyl-5-methyl-5phenyl-4,5-dihydro-1,3,4-oxadiazol-2-yl)methyl)5-chloro-1H-indol-3-yl)-1-(pyridin-4-yl)-1Hpyrazol-4-yl)-3-chloro-1-(4(trifluoro methyl) phenyl)azetidin-2-one (5a) yield $65 \%$, M.P: $158-59^{\circ} \mathrm{C}, \mathrm{IR}$ (KBR) : ('ppm) $3042 \mathrm{~cm}^{-1}(=\mathrm{CH}$ (aromatic), $), 1698 \mathrm{~cm}^{-1} \quad(\mathrm{C}=\mathrm{O})$ $, 1620 \mathrm{~cm}^{-1}(\mathrm{C}=\mathrm{N}), 1140 \mathrm{~cm}^{-1}(\mathrm{~N}-\mathrm{N}), 678 \mathrm{~cm}^{-1}(\mathrm{C}-\mathrm{Cl})$ respectively. ${ }^{1} \mathrm{H}-\mathrm{NMR}\left(400 \mathrm{MHz}, \mathrm{DMSO}-\mathrm{d}_{6}\right) \delta \mathrm{ppm}$ : 8.10(s,1 Hof Pyrazole),7.75-8.43(m,4H of $\left.\mathrm{C}_{5} \mathrm{H}_{4} \mathrm{~N}\right)$ 7.30-7.70(m, $4 \mathrm{H},-\mathrm{CH}$ of indol), $6.80-7.25(\mathrm{~m}, 9 \mathrm{H}$, of $\mathrm{C}_{6} \mathrm{H}_{5}$ and $\left.\mathrm{C}_{6} \mathrm{H}_{4} \mathrm{CF}_{3}\right), 5.45(\mathrm{~d}, 1 \mathrm{H},-\mathrm{CH}$ of aziti din attached to $-\mathrm{Cl}), 5.15(\mathrm{~d}, 1 \mathrm{H},-\mathrm{CH}$ of azitidin ring),3.55(s,2 $2 \mathrm{H},-\mathrm{NCH}_{2}$ attached to indol nucleus), 2.46(s,3H of $-\mathrm{COCH}_{3}$ group), 2.22(s,3H,- $\left.-\mathrm{CH}_{3}\right)$. $\mathrm{C}^{13}$ NMR $400 \mathrm{MHz}$, DMSO-d (' ppm) : 129, 111,121, $126,123,113,135,130,126,129,116,61,62,162$, $143,134,125,132,124,147,114,150,60,159,90$, $169,24,28,142,127,128.5,126.5$ corresponding to $\mathrm{C}_{1}, \mathrm{C}_{2}, \mathrm{C}_{3}, \mathrm{C}_{4}, \mathrm{C}_{5}, \mathrm{C}_{6}, \mathrm{C}_{7}, \mathrm{C}_{8}, \mathrm{C}_{9}, \mathrm{C}_{10}, \mathrm{C}_{11}, \mathrm{C}_{12}, \mathrm{C}_{13}$, $\mathrm{C}_{14}, \mathrm{C}_{15}, \mathrm{C}_{16} \& \mathrm{C}_{20}, \mathrm{C}_{17} \& \mathrm{C}_{19}, \mathrm{C}_{18}, \mathrm{C}_{21,}, \mathrm{C}_{22}, \mathrm{C}_{23} \& \mathrm{C}_{26}, \mathrm{C}_{24}$ $\& \mathrm{C}_{25}, \mathrm{C}_{27}, \mathrm{C}_{28}, \mathrm{C}_{29}, \mathrm{C}_{30}, \mathrm{C}_{31}, \mathrm{C}_{32}, \mathrm{C}_{33}, \mathrm{C}_{34} \& \mathrm{C}_{38}, \mathrm{C}_{35} \& \mathrm{C}_{37}$ and $\mathrm{C}_{36}$ carbon atom respectively. $\operatorname{Mass}(\mathrm{m} / \mathrm{z})$ : 757.16, Anal. Calcd.For $\mathrm{C}_{38} \mathrm{H}_{28} \mathrm{Cl}_{2} \mathrm{~F}_{3} \mathrm{~N}_{7} \mathrm{O}_{3}$ : C, $60.17 \%$; $\mathrm{H}, 3.72 \%$; N, $12.93 \%$; O, 6.33\%. Found: C $60.02 \%, H 3.67 \%, N 12.78 \%$, O $6.17 \%$.

Characterization of 4-(3-(1-((4-acetyl-5-methyl-5(p-tolyl)4,5-dihydro-1,3,4-oxadiazol-2-yl)methyl)5-chloro-1H-indol-3-yl)-1-(pyridin-4-yl)-1Hpyrazol-4-yl)-3-chloro-1-(4-(trifluoro methyl) phenyl)azetidin-2-one (5b)

yield $64 \%$, M.P: $152-53^{\circ} \mathrm{C}$, IR (KBR) :

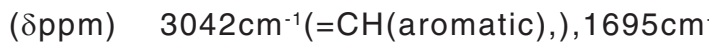
${ }^{1}(\mathrm{C}=\mathrm{O}), 1620 \mathrm{~cm}^{-1}(\mathrm{C}=\mathrm{N}), 1645 \& 1232 \mathrm{~cm}^{-1}(1,3,4-$ oxadiazole), $676 \mathrm{~cm}^{-1}(\mathrm{C}-\mathrm{Cl})$ respectively. ${ }^{1} \mathrm{H}-\mathrm{NMR}$ $\left(400 \mathrm{MHz}, \mathrm{DMSO}-\mathrm{d}_{6}\right) \delta \mathrm{ppm}: 8.09(\mathrm{~s}, 1 \mathrm{H}, \mathrm{N}-\mathrm{CH}$ gp.),7.75-8.41(m,4 $\left.\mathrm{Hof}^{\mathrm{C}_{5}} \mathrm{H}_{4} \mathrm{~N}\right), 7.30-7.70(\mathrm{~m}, 4 \mathrm{H},-\mathrm{CH}$ of indol), $6.85-7.15\left(\mathrm{~m}, 8 \mathrm{H}\right.$, of $-\mathrm{C}_{6} \mathrm{H}_{4}$ and $\left.\mathrm{C}_{6} \mathrm{H}_{4} \mathrm{CF}_{3}\right)$ , $5.44(\mathrm{~d}, 1 \mathrm{H},-\mathrm{CH}$ of azitidin attached to $-\mathrm{Cl})$, $5.13\left(\mathrm{~d}, 1 \mathrm{H},-\mathrm{CH}\right.$ of azitidin ring), $3.52\left(\mathrm{~s}, 2 \mathrm{H},-\mathrm{NCH}_{2}\right.$ attached to indolnucleus ), 2.45 (s,3 $3 \mathrm{H}$ of $-\mathrm{COCH}_{3}$ group), $2.23\left(\mathrm{~s}, 3 \mathrm{H}, \mathrm{CH}_{3}\right), 1.57\left(\mathrm{~s}, 3 \mathrm{H},-\mathrm{CH}_{3}\right.$ attached to phenyl ring).. $\mathrm{C}^{13}-\mathrm{NMR} \quad 400 \mathrm{MHz}$ DMSO-d ${ }_{6}$ (' ppm) : 129.1, 111.2, 121.3, 125.9, 123, 112.7, 134.7, 130, 126, 129, 115.8,61, 62, $162.1,142.8,134,125.1$, $132,124,147,114,150,60,158.2,90,168.5,24$, $28,139,127,128.8,136.5,21.21 .5$ corresponding to $\mathrm{C}_{1}, \mathrm{C}_{2}, \mathrm{C}_{3}, \mathrm{C}_{4}, \mathrm{C}_{5}, \mathrm{C}_{6}, \mathrm{C}_{7}, \mathrm{C}_{8}, \mathrm{C}_{9}, \mathrm{C}_{10}, \mathrm{C}_{11}, \mathrm{C}_{12}, \mathrm{C}_{13}, \mathrm{C}_{14}$, $\mathrm{C}_{15}, \mathrm{C}_{16} \& \mathrm{C}_{20}, \mathrm{C}_{17} \& \mathrm{C}_{19}, \mathrm{C}_{18}, \mathrm{C}_{21}, \mathrm{C}_{22}, \mathrm{C}_{23} \& \mathrm{C}_{26}, \mathrm{C}_{24} \& \mathrm{C}_{25}$, $\mathrm{C}_{27}, \mathrm{C}_{28}, \mathrm{C}_{29}, \mathrm{C}_{30}, \mathrm{C}_{31}, \mathrm{C}_{32}, \mathrm{C}_{33}, \mathrm{C}_{34} \& \mathrm{C}_{38}, \mathrm{C}_{35} \& \mathrm{C}_{37}, \mathrm{C}_{36}$ and $\mathrm{C}_{39}$ carbon atom respectively. Mass $(\mathrm{m} / \mathrm{z}): 771.17$ , Anal. Calcd. For $\mathrm{C}_{39} \mathrm{H}_{30} \mathrm{Cl}_{2} \mathrm{~F}_{3} \mathrm{~N}_{7} \mathrm{O}_{3}: \mathrm{C}, 60.63 \% ; \mathrm{H}$, $3.91 \%$; N $12.69 \%$; O, $6.21 \%$. Found: C $60.47 \%, \mathrm{H}$ $3.76 \%, \mathrm{~N} 12.54 \%$, O $6.04 \%$

Characterization of 4-(3-(1-((4-acetyl-5-(4methoxyphenyl)-5-methyl-4,5-dihydro-1,3,4oxadiazol-2-yl)methyl)-5-chloro-1H-indol-3-yl)-1(pyridin-4-yl)-1H-pyrazol-4-yl)-3-chloro-1-(4(trifluoromethyl)phenyl) azetidin-2-one (5c)

Yield $62 \%$, M.P: $142-44^{\circ} \mathrm{C}$, IR (KBR) : (8ppm) $3042 \mathrm{~cm}^{-1}(=\mathrm{CH}($ aromatic $)),, 1680 \mathrm{~cm}^{-}$ ${ }^{1}(\mathrm{C}=\mathrm{O}), 1617 \mathrm{~cm}^{-1}(\mathrm{C}=\mathrm{N}), 1645 \& 1232 \mathrm{~cm}^{-1}(1,3,4-$ oxadiazole), $675 \mathrm{~cm}^{-1}(\mathrm{C}-\mathrm{Cl})$ respectively. ${ }^{1} \mathrm{H}-\mathrm{NMR}$ $\left(400 \mathrm{MHz}, \mathrm{DMSO}-\mathrm{d}_{6}\right) \delta \mathrm{ppm}: 8.07(\mathrm{~s}, 1 \mathrm{H}, \mathrm{N}-\mathrm{CH}$ gp.),7.75-8.40(m, $\left.4 \mathrm{H}_{\text {of }} \mathrm{C}_{6} \mathrm{H}_{4} \mathrm{~N}\right), 7.30-7.70(\mathrm{~m}, 4 \mathrm{H},-\mathrm{CH}$ of indol), $6.80-7.16\left(\mathrm{~m}, 8 \mathrm{H}\right.$, of $-\mathrm{C}_{6} \mathrm{H}_{4}$ and $\left.\mathrm{C}_{6} \mathrm{H}_{4} \mathrm{CF}_{3}\right)$, $5.43(\mathrm{~d}, 1 \mathrm{H},-\mathrm{CH}$ of azitidin attached to $-\mathrm{Cl})$, $5.12\left(\mathrm{~d}, 1 \mathrm{H},-\mathrm{CH}\right.$ of azitidin ring), $3.50\left(\mathrm{~s}, 2 \mathrm{H},-\mathrm{NCH}_{2}\right.$ attached to indol nucleus), $2.46(\mathrm{~s}, 3 \mathrm{H}$ of $\mathrm{COCH}_{3}$ group), 2.24(s, $\left.3 \mathrm{H},-\mathrm{CH}_{3}\right), 3.82\left(\mathrm{~s}, 3 \mathrm{H},-\mathrm{OCH}_{3}\right)$. 
$\mathrm{C}^{13}-\mathrm{NMR}$ 400MHz DMSO-d $(' \mathrm{ppm}): 129.2,111$, $121.9,125.5,122.6,113,134.5,130,125.5,129,116$ $61,62,162.5,143,133.9,125.5,132,124.3,147$ $114,150,58,160,91,169,23.9,28,135,128,114.5$ 158.7, 55.8 corresponding to $\mathrm{C}_{1}, \mathrm{C}_{2}, \mathrm{C}_{3}, \mathrm{C}_{4}, \mathrm{C}_{5}, \mathrm{C}_{6}$, $\mathrm{C}_{7}, \mathrm{C}_{8}, \mathrm{C}_{9}, \mathrm{C}_{10}, \mathrm{C}_{11}, \mathrm{C}_{12}, \mathrm{C}_{13}, \mathrm{C}_{14}, \mathrm{C}_{15}, \mathrm{C}_{16} \& \mathrm{C}_{20}, \mathrm{C}_{17} \& \mathrm{C}_{19}$, $\mathrm{C}_{18}, \mathrm{C}_{21}, \mathrm{C}_{22}, \mathrm{C}_{23} \& \mathrm{C}_{26}, \mathrm{C}_{24} \& \mathrm{C}_{25}, \mathrm{C}_{27}, \mathrm{C}_{28}, \mathrm{C}_{29}, \mathrm{C}_{30}, \mathrm{C}_{31}$, $\mathrm{C}_{32}, \mathrm{C}_{33}, \mathrm{C}_{34} \& \mathrm{C}_{38}, \mathrm{C}_{35} \& \mathrm{C}_{37}, \mathrm{C}_{36}$ and $\mathrm{C}_{39}$ carbon atom respectively. Mass(m/z) : 787.17 , Anal.Calcd.For $\mathrm{C}_{39} \mathrm{H}_{30} \mathrm{Cl}_{2} \mathrm{~F}_{3} \mathrm{~N}_{7} \mathrm{O}_{4}$ : C, 59.40\%; H, 3.83\%; N, 12.43\%; O, 8.12\%. Found: C 59.24\%, H 3.67\%, N 12.28\%, O $7.98 \%$.

Characterization of 4-(3-(1-)((4-acetyl-5-(4chlorophenyl)-5-methyl-4,5-dihydro-1,3,4oxadiazol-2-yl)methyl)-5-chloro-1H-indol-3-yl)-1(pyridin-4-yl)-1H-pyrazol-4-yl)-3-chloro-1-(4(trifluoromethyl) phenyl)azetidin-2-one (5d) Yield $66 \%$, M.P: $165-67^{\circ} \mathrm{C}$, IR (KBR) :

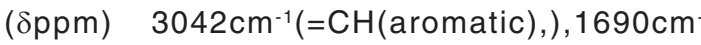
${ }^{1}(\mathrm{C}=\mathrm{O}), 1623 \mathrm{~cm}^{-1}(\mathrm{C}=\mathrm{N}), 1645 \& 1232 \mathrm{~cm}^{-1}(1,3,4-$ oxadiazole), $677 \mathrm{~cm}^{-1}(\mathrm{C}-\mathrm{Cl})$ respectively. ${ }^{1} \mathrm{H}-\mathrm{NMR}$ (400MHz, DMSO- $\mathrm{d}_{6}$ ) $\delta$ ppm: 8.08(s, $1 \mathrm{H}, \mathrm{N}-\mathrm{CH}$

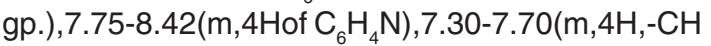
of indol), $6.85-7.18\left(\mathrm{~m}, 8 \mathrm{H}\right.$, of $-\mathrm{C}_{6} \mathrm{H}_{4} \mathrm{Cl}$ and $\left.\mathrm{C}_{6} \mathrm{H}_{4} \mathrm{CF}_{3}\right), 5.43(\mathrm{~d}, 1 \mathrm{H},-\mathrm{CH}$ of azitidin attached to $\mathrm{Cl}), 5.13(\mathrm{~d}, 1 \mathrm{H},-\mathrm{CH}$ of azitidin ring $), 3.57\left(\mathrm{~s}, 2 \mathrm{H},-\mathrm{NCH}_{2}\right.$ attached to indol nucleus), 2.47(s,3H of $\mathrm{COCH}_{3}$ group), 2.24(s,3H,-CH $)_{3}$. C ${ }^{13}-\mathrm{NMR} 400 \mathrm{MHz}$ DMSO$\mathrm{d}_{6}$ (' ppm) : 129.2, 111.3, 121.7, 125.6, 122.4, 112.4, $134.5,130.3,125.4,129,115.9,60.9,62.1,162.3$, $142.8,133.9,125.4,124.1,146.9,113.9,149.9$, $60,158.3,90.2,168.5,23.7,27.9,140.7$, $125.4,128.7,132$.3corresponding to $\mathrm{C}_{1}, \mathrm{C}_{2}, \mathrm{C}_{3}, \mathrm{C}_{4}$, $\mathrm{C}_{5}, \mathrm{C}_{6}, \mathrm{C}_{7}, \mathrm{C}_{8}, \mathrm{C}_{9}, \mathrm{C}_{10}, \mathrm{C}_{11}, \mathrm{C}_{12}, \mathrm{C}_{13}, \mathrm{C}_{14}, \mathrm{C}_{15}, \mathrm{C}_{16} \& \mathrm{C}_{20}$, $\mathrm{C}_{17} \& \mathrm{C}_{19}, \mathrm{C}_{18}, \mathrm{C}_{21}, \mathrm{C}_{22}, \mathrm{C}_{23} \& \mathrm{C}_{26}, \mathrm{C}_{24} \& \mathrm{C}_{25}, \mathrm{C}_{27}, \mathrm{C}_{28}, \mathrm{C}_{29}$, $\mathrm{C}_{30}, \mathrm{C}_{31}, \mathrm{C}_{32}, \mathrm{C}_{33}, \mathrm{C}_{34} \& \mathrm{C}_{38}, \mathrm{C}_{35} \& \mathrm{C}_{37}$ and $\mathrm{C}_{36}$ carbon atom respectively. Mass $(\mathrm{m} / \mathrm{z}): 791.12$, Anal.Calcd.For $\mathrm{C}_{38} \mathrm{H}_{27} \mathrm{Cl}_{3} \mathrm{~F}_{3} \mathrm{~N}_{7} \mathrm{O}_{3}$ : C, $57.55 \% ; \mathrm{H}$, $3.43 \%$; N,12.36\%; O,6.05\% .Found: C $57.40 \%$, H $3.28 \%$, N $12.21 \%$, O $5.90 \%$

Characterization of 4-(3-(1-)((4-acetyl-5-(4bromophenyl)-5-methyl-4,5-dihydro-1,3,4oxadiazol-2-yl)methyl)-5-chloro-1H-indol-3-yl)-1(pyridin-4-yl)-1H-pyrazol-4-yl)-3-chloro-1-(4(trifluoromethyl) phenyl)azetidin-2-one (5e)

Yield $67 \%$, M.P: $162-63^{\circ} \mathrm{C}$, IR (KBR) :

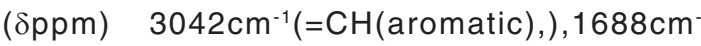

${ }^{1}(\mathrm{C}=\mathrm{O}), 1623 \mathrm{~cm}^{-1}(\mathrm{C}=\mathrm{N}), 1645 \& 1232 \mathrm{~cm}^{-1}(1,3,4-$ oxadiazole), $676 \mathrm{~cm}^{-1}(\mathrm{C}-\mathrm{Cl})$ respectively. ${ }^{1} \mathrm{H}-\mathrm{NMR}$ (400MHz, DMSO-d ${ }_{6}$ ) $\delta p p m: 8.09(\mathrm{~s}, 1 \mathrm{H}, \mathrm{N}-\mathrm{CH}$ gp.),7.75-8.42(m,4 $\left.\mathrm{Hof} \mathrm{C}_{6} \mathrm{H}_{4} \mathrm{~N}\right), 7.30-7.70(\mathrm{~m}, 4 \mathrm{H},-\mathrm{CH}$ of indol), $6.80-7.20\left(\mathrm{~m}, 8 \mathrm{H}\right.$, of $-\mathrm{C}_{6} \mathrm{H}_{4} \mathrm{Br}$ and $\left.\mathrm{C}_{6} \mathrm{H}_{4} \mathrm{CF}_{3}\right), 5.42(\mathrm{~d}, 1 \mathrm{H},-\mathrm{CH}$ of azitidin attached to $\mathrm{Cl}), 5.12(\mathrm{~d}, 1 \mathrm{H},-\mathrm{CH}$ of azitidin ring $), 3.58\left(\mathrm{~s}, 2 \mathrm{H},-\mathrm{NCH}_{2}\right.$ attached to indol nucleus), $2.46\left(\mathrm{~s}, 3 \mathrm{H}\right.$ of $-\mathrm{COCH}_{3}$ group), 2.23(s,3H,-CH $)_{3}$. $\mathrm{C}^{13}-\mathrm{NMR} 400 \mathrm{MHz}$, DMSO$\mathrm{d}_{6}(\delta \mathrm{ppm}): 129,111.2,121.6,125.6,122.4,112.5$, $134.5,130.2$, 125.5, 129,115.9 ,60.9, 62, 162.3, $142.8,133.8,125.3,132.2,124,147,114,150$, 59,158, 90.2,168.5, 23.9,28, 141.7, 129.2, 131.5, 121.2corresponding to $\mathrm{C}_{1}, \mathrm{C}_{2}, \mathrm{C}_{3}, \mathrm{C}_{4}, \mathrm{C}_{5}, \mathrm{C}_{6}, \mathrm{C}_{7}, \mathrm{C}_{8}$, $\mathrm{C}_{9}, \mathrm{C}_{10}, \mathrm{C}_{11}, \mathrm{C}_{12}, \mathrm{C}_{13}, \mathrm{C}_{14}, \mathrm{C}_{15}, \mathrm{C}_{16} \& \mathrm{C}_{20}, \mathrm{C}_{17} \& \mathrm{C}_{19}, \mathrm{C}_{18}$, $\mathrm{C}_{21}, \mathrm{C}_{22}, \mathrm{C}_{23} \& \mathrm{C}_{26}, \mathrm{C}_{24} \& \mathrm{C}_{25}, \mathrm{C}_{27}, \mathrm{C}_{28}, \mathrm{C}_{29}, \mathrm{C}_{30}, \mathrm{C}_{31}$, $\mathrm{C}_{32}, \mathrm{C}_{33}, \mathrm{C}_{34} \& \mathrm{C}_{38}, \mathrm{C}_{35} \& \mathrm{C}_{37}$ and $\mathrm{C}_{36}$ carbon atom respectively. Mass(m/z) : 835.07 , Anal.Calcd.For $\mathrm{C}_{38} \mathrm{H}_{27} \mathrm{BrCl}_{2} \mathrm{~F}_{3} \mathrm{~N}_{7} \mathrm{O}_{3}: \mathrm{C}, 54.50 \% ; \mathrm{H}, 3.25 \% ; \mathrm{N}, 11.71 \%$; O, $5.73 \%$.Found: C 54.35\%, H 3.10\%, N 11.56\%, O $5.57 \%$.

Characterization of 4-(3-(1-((4-acetyl-5-methyl5-(4-nitrophenyl)-4,5-dihydro-1,3,4 oxadiazol -2yl)methyl)-5-chloro-1H-indol-3-yl)-1-(pyridin-4yl)-1H-pyrazol-4-yl)-3-chloro -1-(4-(trifluoro methyl)phenyl)azetidin-2-one (5f)

yield $70 \%$, M.P: $184-86^{\circ} \mathrm{C}$, IR (KBR) : ('ppm) $3042 \mathrm{~cm}^{-1}(=\mathrm{CH}$ (aromatic), ), $1697 \mathrm{~cm}$. ${ }^{1}(\mathrm{C}=\mathrm{O}), 1625 \mathrm{~cm}^{-1}(\mathrm{C}=\mathrm{N}), 1645 \& 1232 \mathrm{~cm}^{-1}(1,3,4-$ oxadiazole), $678 \mathrm{~cm}^{-1}(\mathrm{C}-\mathrm{Cl})$ respectively. ${ }^{1} \mathrm{H}-\mathrm{NMR}$ (400MHz, DMSO-d $\left.{ }_{6}\right) \delta p p m: 8.10(\mathrm{~s}, 1 \mathrm{H}, \mathrm{N}-\mathrm{CH}$

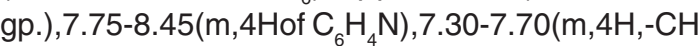
of indol), 6.95-7.28(m, $8 \mathrm{H}$, of $-\mathrm{C}_{6} \mathrm{H}_{4} \mathrm{NO}_{2}$ and $\left.\mathrm{C}_{6} \mathrm{H}_{4} \mathrm{CF}_{3}\right), 5.44(\mathrm{~d}, 1 \mathrm{H},-\mathrm{CH}$ of azitidin attached to $\mathrm{Cl}), 5.14\left(\mathrm{~d}, 1 \mathrm{H},-\mathrm{CH}\right.$ of azitidin ring),3.71(s,2H,- $\mathrm{NCH}_{2}$ attached to indol nucleus), 2.46 (s,3H of $\mathrm{COCH}_{3}$ group) , 2.20(s,3H,- $\left.\mathrm{CH}_{3}\right) . \quad \mathrm{C}^{13}-\mathrm{NMR} 400 \mathrm{MHz}$ , DMSO-d ( $_{6}$ ppm ): 129.2, 111.3, 121.7, 125.7, $122.5,112.5,134.6,130.2,125.4,129,115.8,61$, $62.1,162.2,142.8,133.8,125.3,132.2,124.2,146.9$, $113.9,149.9,60,158.2,90.2,168.7,23.8,27.9$, $148.7,127.8,123.7,145.9$ corresponding to $C_{1}, C_{2}$, $\mathrm{C}_{3}, \mathrm{C}_{4}, \mathrm{C}_{5}, \mathrm{C}_{6}, \mathrm{C}_{7}, \mathrm{C}_{8}, \mathrm{C}_{9}, \mathrm{C}_{10}, \mathrm{C}_{11}, \mathrm{C}_{12}, \mathrm{C}_{13}, \mathrm{C}_{14}, \mathrm{C}_{15}$, $\mathrm{C}_{16} \& \mathrm{C}_{20}, \mathrm{C}_{17} \& \mathrm{C}_{19}, \mathrm{C}_{18}, \mathrm{C}_{21}, \mathrm{C}_{22}, \mathrm{C}_{23} \& \mathrm{C}_{26}, \mathrm{C}_{24} \& \mathrm{C}_{25}$, $\mathrm{C}_{27}, \mathrm{C}_{28}, \mathrm{C}_{29}, \mathrm{C}_{30}, \mathrm{C}_{31}, \mathrm{C}_{32}, \mathrm{C}_{33}, \mathrm{C}_{34} \& \mathrm{C}_{38}, \mathrm{C}_{35} \& \mathrm{C}_{37}$ and $\mathrm{C}_{36}$ carbon atom respectively. Mass $(\mathrm{m} / \mathrm{z}): 802.14$, Anal.Calcd.For $\mathrm{C}_{38} \mathrm{H}_{27} \mathrm{Cl}_{2} \mathrm{~F}_{3} \mathrm{~N}_{8} \mathrm{O}_{5}: \mathrm{C}, 56.80 \% ; \mathrm{H}$, $3.39 \%$; N, 13.94\%; O, 9.96\%. Found: C 56.65\%, H $3.23 \%$, N $13.77 \%$, O $9.80 \%$. 
Characterization of 4-(3-(1-((4-acetyl-5-methyl-5(4-(trifluoromethyl)phenyl)-4,5-dihydro-1,3,4oxadiazol-2-yl)methyl)-5-chloro-1H-indol-3-yl)-1(pyridin-4-yl)-1H-pyrazol-4-yl)-3-chloro-1-(4(trifluoromethyl) phenyl)azetidin-2-one(5g)

Yield $68 \%$, M.P: $178-79{ }^{\circ} \mathrm{C}$, IR (KBR) :

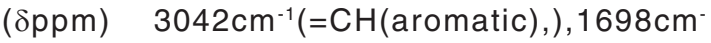
${ }^{1}(\mathrm{C}=\mathrm{O}), 1624 \mathrm{~cm}^{-1}(\mathrm{C}=\mathrm{N}), 1645 \& 1232 \mathrm{~cm}^{-1}(1,3,4-$ oxadiazole), $677 \mathrm{~cm}^{-1}(\mathrm{C}-\mathrm{Cl})$ respectively. ${ }^{1} \mathrm{H}-\mathrm{NMR}$ $\left(400 \mathrm{MHz}\right.$, DMSO-d $\left.{ }_{6}\right) \delta p p m: 8.08(\mathrm{~s}, 1 \mathrm{H}, \mathrm{N}-\mathrm{CH}$ gp.),7.75-8.44(m,4 Hof $\left.\mathrm{C}_{6} \mathrm{H}_{4} \mathrm{~N}\right), 7.30-7.70(\mathrm{~m}, 4 \mathrm{H},-\mathrm{CH}$ of indol), $6.90-7.25\left(\mathrm{~m}, 8 \mathrm{H}\right.$, of two $-\mathrm{C}_{6} \mathrm{H}_{4} \mathrm{CF}_{3}$ rings), $5.45(\mathrm{~d}, 1 \mathrm{H},-\mathrm{CH}$ of azitidin attached to $-\mathrm{Cl})$, $5.14(\mathrm{~d}, 1 \mathrm{H},-\mathrm{CH}$ of azitidin ring $), 3.68\left(\mathrm{~s}, 2 \mathrm{H},-\mathrm{NCH}_{2}\right.$ attached to indol nucleus), 2.47(, $3 \mathrm{H}$ of $\mathrm{COCH}_{3}$ group), 2.18(s,3H,-CH $\mathrm{CH}_{3} \cdot \mathrm{C}^{13}-\mathrm{NMR} 400 \mathrm{MHz}$ ,DMSO-d 6 (8ppm): 129.3, 111.4, 121.7, 125.5, 122.4, $112.5,134.6,130.2,125.4,129,115.8,60.9,62$, $162.2,142.8,133.8,125.3,132.1,124.1,146.9$, $113.9,149.9,60,158.2,90.2,168.5,23.7,27.9$, $145.9,127.2,124.9,129,124.2$ corresponding to $\mathrm{C}_{1}, \mathrm{C}_{2}, \mathrm{C}_{3}, \mathrm{C}_{4}, \mathrm{C}_{5}, \mathrm{C}_{6}, \mathrm{C}_{7}, \mathrm{C}_{8}, \mathrm{C}_{9}, \mathrm{C}_{10}, \mathrm{C}_{11}, \mathrm{C}_{12}, \mathrm{C}_{13}$, $\mathrm{C}_{14}, \mathrm{C}_{15}, \mathrm{C}_{16} \& \mathrm{C}_{20}, \mathrm{C}_{17} \& \mathrm{C}_{19}, \mathrm{C}_{18}, \mathrm{C}_{21}, \mathrm{C}_{22}, \mathrm{C}_{23} \& \mathrm{C}_{26}, \mathrm{C}_{24}$ $\& \mathrm{C}_{25}, \mathrm{C}_{27}, \mathrm{C}_{28}, \mathrm{C}_{29}, \mathrm{C}_{30}, \mathrm{C}_{31}, \mathrm{C}_{32}, \mathrm{C}_{33}, \mathrm{C}_{34} \& \mathrm{C}_{38}, \mathrm{C}_{35}$ $\& \mathrm{C}_{37} \mathrm{C}_{36}$ and $\mathrm{C}_{39}$ carbonatom respectively. Mass $(\mathrm{m} /$ z):825.15 , Anal. Calcd. For $\mathrm{C}_{38} \mathrm{H}_{27} \mathrm{Cl}_{2} \mathrm{~F}_{6} \mathrm{~N}_{7} \mathrm{O}_{3}: \mathrm{C}$, $56.67 \%$; H, 3.29\%; N, 11.86\%; O, 5.81\%. Found: C $56.52 \%$, H $3.13 \%$, N $11.70 \%$, O $5.65 \%$.

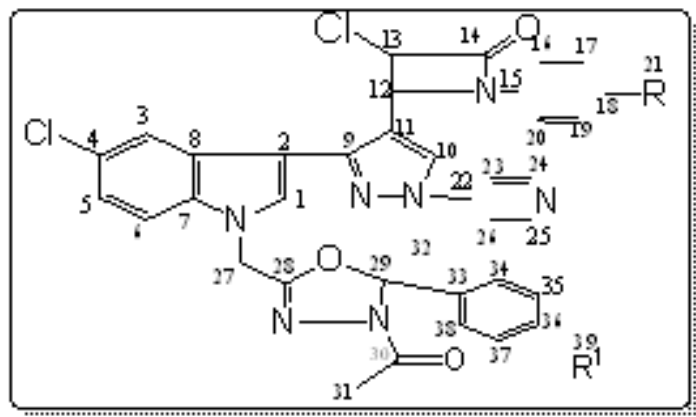

\section{Biological activity}

The newly synthesized compounds 4-(3(1-((4-acetyl-5-methyl-5-(p-substituted phenyl)-4,5dihydro-1,3,4-oxadiazol-2-yl)methyl)-5-chloro-1Hindol-3-yl)-1-(pyridin-4-yl)-1H-pyrazol-4 -yl)-3chloro-1-(4-substituted phenyl)azetidin-2-one (5ag), were screened for their antimicrobial studies against antibacterial and antifungal activity by Disc Diffusion method ${ }^{22}$. The synthesized compounds were used at the concentration of $2501 / 4 \mathrm{~g} / \mathrm{ml}$ and $5001 / 4 \mathrm{~g} / \mathrm{ml}$ using DMF as a solvent ${ }^{23}$. The amoxicillin 10 1/4 g/disc and cefaclor 30 1/4 g/disc were used as a standard .Whatman No.1 filter paper disk of $5 \mathrm{~mm}$ diameter were sterile nutrient agar at $45^{\circ} \mathrm{C}$.

The sterile disks were impregnated with different compounds synthesized compounds $(2501 / 4 \mathrm{~g} / \mathrm{ml})$. The impregnated disks were placed on the medium suitably spaced apart and the plates were incubated at $25^{\circ} \mathrm{C}$ for $1 \mathrm{~h}$. To permit good diffusion and then transferred to an incubator at 37 ${ }^{\circ} \mathrm{C}$ for $48 \mathrm{~h}$.for bacteria, and at $28{ }^{\circ} \mathrm{C}$ for $72 \mathrm{~h}$. For yeast and fungi. The incubation zones aused by the various compounds on the microorganisms were examined. The results of the preliminary screening test are listed in table-3.

\section{Antibacterial activity}

The antibacterial activity of $5(\mathrm{a}-\mathrm{g})$ were screened against the Staphylococus aureus (gram positive), Bacillus cerus, Escherichia coli (gram negative) and Pseudomonas aeruginosa organisms. In a given series of compounds having nitro (5f) and trifluoromethyl (5g) exhibit high bacterialactivity ${ }^{24,25}$ when compared to other substituents. The structural activity relationship for different substituents is in the order i.e. $-\mathrm{NO}_{2}>-\mathrm{CF}_{3}$ $>-\mathrm{Cl}>-\mathrm{Br}>-\mathrm{H}>-\mathrm{CH}_{3}>-\mathrm{OCH}_{3}$. Here amoxicillin and cefaclor are tested as reference compounds to compare the activity. The antibacterial activity of 5(ag) was shown in the below given table.

\section{Antifungal activity}

The antifungal activity of final compounds 5(a-g) were screened aginst aspergillus niger ,Candida albicans . In a given series of compounds containing trifluoro methyl and nitro groups in their structures has shown increased effect on their antifungal activity . The structural activity relationship for different substituents is in the order i.e. $-\mathrm{NO}_{2}>-$ $\mathrm{CF}_{3}>-\mathrm{Cl}>-\mathrm{Br}>-\mathrm{H}>-\mathrm{CH}_{3}>-\mathrm{OCH}_{3}$ Here ketoconazole is tested as reference compound to compare the antifungal activity. Antifungal activity of 4-(3-(1-((4-acetyl-5-methyl-5-(p-substituted phenyl)-4,5-dihydro-1,3,4-oxadiazol-2-yl)methyl)5chloro-1H-indol-3-yl)-1-(pyridin-4-yl)-1H-pyrazol4-yl)-3-chloro-1-(4-substituted phenyl) azetidin -2one $(5 \mathrm{a}-\mathrm{g})$ was shown in the below given table. 
Table 3:

\begin{tabular}{|c|c|c|c|c|c|c|c|}
\hline \multirow{3}{*}{$\begin{array}{l}\text { S. } \\
\text { No. }\end{array}$} & \multirow[t]{3}{*}{ Compd. } & \multicolumn{6}{|c|}{ Zone of Inhibition (mm) } \\
\hline & & \multicolumn{3}{|c|}{ Anti bacterial activity } & \multicolumn{3}{|c|}{ Anti fungal activity } \\
\hline & & $\begin{array}{c}\text { Staphylococus } \\
\text { aureus } \\
\text { NCCS } \\
2079\end{array}$ & $\begin{array}{l}\text { Bacillus } \\
\text { cereus } \\
\text { NCCS } \\
2106\end{array}$ & $\begin{array}{c}\text { Escherichia } \\
\text { Coli } \\
\text { NCCS } \\
2065\end{array}$ & $\begin{array}{c}\text { Pseudomonas } \\
\text { aeruginosa } \\
\text { NCCS } \\
2200\end{array}$ & $\begin{array}{c}\text { Aspergillus } \\
\text { niger } \\
\text { NCCS } \\
1196\end{array}$ & $\begin{array}{c}\text { Candida } \\
\text { albicans } \\
\text { NCCS } \\
3471\end{array}$ \\
\hline 1) & $5 a$ & 11 & 10 & 11 & 11 & 13 & 15 \\
\hline 2) & $5 b$ & 10 & 09 & 10 & 09 & 12 & 13 \\
\hline 3) & $5 c$ & 08 & 08 & 09 & 08 & 11 & 11 \\
\hline 4) & $5 d$ & 13 & 12 & 11 & 13 & 14 & 19 \\
\hline 5) & $5 e$ & 12 & 11 & 10 & 12 & 13 & 16 \\
\hline 6) & $5 f$ & 17 & 16 & 15 & 16 & 19 & 21 \\
\hline 7) & $5 g$ & 16 & 14 & 13 & 15 & 18 & 20 \\
\hline 8) & Amoxicillin & 21 & 27 & 24 & 22 & - & \\
\hline 9) & Cefaclor & 19 & 22 & 19 & 20 & - & - \\
\hline 10) & Ketoconazol & - & - & - & - & 23 & 26 \\
\hline
\end{tabular}

\section{CONCLUSION}

Indol bearing pyrazole ring, besides azitidinone moiety and the 1,3,4-oxadiazole group were prepared by acetic anhydride reaction with acetohydrazid group. These synthons were purified \& charecterized by chromatographic and spectral techniques. Indol derivatives were subjected to antimicrobial evaluation and some of these compounds were found to posses good anti bacterial and anti microbial activity.

\section{ACKNOWLEDGEMENTS}

The author (P.Ashokgajapathiraju) thanks to U G C-S A P and U G C-B S R, New Delhi for financial assistance and also thankful to IICT Hyderabad and CDRI Lucknow for spectral and analytical data. I express my sincere thanks to my research Supervisor Prof. J.Sreeramulu for his valuable guidance.

\section{REFERENCES}

1. Walser, A.; Flynn, T.; Mason, C. J. Heterocyclic. Chem. 1991, 28 , 1121-1125.

2. Ewiss, N.F. ; Bahajaj, A.A. ; Elsherbini, E.A. j. Heterocyclic chem. 1986, 23,1451-1458.

3. Bhat, A.R.; Bhat, G.V.; Shenoy, G.G. J. Pharm. Pharmacol. 2001, 53, 267-272.

4. Todoulou, O.G.; Papadaki-Valaraki, A.E.; Flippatos, E.C.; Ilkeda, S.; De Clercq, E. Eur.J.Med. Chem.. 1994 , 29 , 127-131.

5. Holla, B.S.; Gonsalves, R.; Shenoy, S.; Eur. J. Med. Chem. 2000, 35 , 267-271 Chem Inform Abstract: Synthesis and Antibacterial Studies of a New Series of 1,2-Bis(1,3,4oxadiazol-2-yl)ethanes and 1,2-Bis(4amino-1,2,4-triazol-3-yl)ethanes.
6. Barbucenu,S.F.; Bancescu, G.; Cretu,O.D.; Draghici,C.; Bancescu,A.;Radu-Popescu.M. Rev. Chem. (Bucuresti).61.Nr.2., 2010, 140145.

7. Kumar, G.V.S.; Rajendraprasad, Y.; Mallikarjuna, B.P.; Chandrashekar,S.M.; Kistayya,C. Eur. J. Med. Chem., 2010, 45, 2063-2074.

8. Shirote, P.J. ; Bhatia, M.S. Arab. J. Chem., 2010, doi: 10.1016/j.ara bjc.2010.07.004.

9. Parkash,O.; Kumar, M.; Sharma,C.; Aneja. K.R.;Eur. J. Med. Chem., 2010,doi:10.1016/ j.ejmech.2010.06.023.

10. Jou, X.Z.; Lai, L.H.; Jin, G.Y. J.Agrc. Food.Chem. 2002, 50, 3757-3760. 
11. Padmavathi,V.; Reddy,G.S.; Padmaja,A.; Kondaiah,P.; Ali-Shazia. Eur. J. Med. Chem., 2009, 44, 2106-2112.

12. Akhter, M.; Husain, A.; Azad,B.; Ajmal,M.; Eur. J. Med. Chem., 2009, 44, 2372-2378.

13. Idrees, G.A.; Aly, O.M.; El-Din, G.; AbuoRahma, A.A.; Shazia, M.F.R. Eur. J. Med. Chem., 2009, 44, 3973-3980.

14. Palaska, E.; Sahin,G.; Kelicen,P.;Durlu,N.T.; Altinok,G. II Farmaco 2002 , 57, 101-107.

15. Amir, M.; Shikha, K.; Eur.J. Med. Chem. 2004, 39, 535-545.

16. Jayashankar,B.; Rai, K.M.L.;Baskaran,N.; Shazia.H.S.S. Eur. J. Med. Chem., 2009, 44, 3898-3902

17. Kumar, D.; Sundaree, S.; Johnson E.O.; Shah, K. Bioorg. Med. Chem. Lett., 2009, 19, 44924494

18. Bhat, J.J.; Shah, B.R.; Shah,H.P.; Trivedi, P.B.; Undavia, N.K.; Desai, N.C. Indian j. chem. 1994 , 33b , 189-192

19. Shashikan, D.; Bhandari,V.; Bothara, K.G.;
Raut,M.K.; Patil, A.A.; Sarkate, A.P.; and Mokale, V.J. Bioorg. Med. Chem. Lett., 2008, 16, 1822-1831

20. Macaev, F.; Rusu, G.; Pogervnoi, S.; Gudima, A.; Stingasi, E.; Vlad, L. et.al Bio org. Med. Chem. 2005, 13, 4842-4850

21. Zargahi, A.; Sayyed, A.; Tabatabai, M.; Faizi, A.; Ahadian, P.; Navavi,V.; Zanganeh,A.; Shafiee, Biorg. Med.Chem. 2005 , 15, 18631865

22. G. Nagalakshmi; Indian Journal of Pharmaceutical Science, 2008, plaintiff. 4955.

23. Sudhir Bharadwaj.; Bharat Parashar.; NarendraParashar and Sharma,V.K.; Scholar Research Library, Achieves of Applied Science Research, 2011, 3(2), 558-567.

24. Colle, J G.; Duguid, J P.; Fraser, A G.; and Mammion, B P. "Mackie and McCartney practical Medical Microbiology", Churchil, Livingston Ltd, London, 13th ed ,Vol.2. 1989.

25. Irfan Ali Mohammed, Der Pharmacia Sinica, 2011, 2(6), 102-106. 